\title{
Research of Protective Coatings Application on Polymer Formulations Made by Additive Technology
}

\author{
Josip GROŠ*, Pero RAOS, Mirela LESKOVEC
}

\begin{abstract}
Injection molding of polymer moldings has proven to be a fast way of making products for medium and high series production for many years. In order to produce a quality product, all stages of the process must be under control (tools, materials, injection molding machine, etc.). In the last two decades, additive technology for the production of prototypes and, more recently, finished products has been developing intensively. The biggest disadvantage of additive technology is the limited choice of polymer materials and different mechanical properties of products compared to products produced by injection molding technology. Making a mold for the injection molding process is time consuming and expensive, and for smaller batches, an unprofitable process. By making molds for injection molding with additive technology, we accelerate the process of production of finished products. One of the disadvantages of making molds in this way is their durability. In order to increase the durability of the mold, the area of coating application will be investigated. The research was conducted as part of the application for a doctoral thesis. The paper will be divided into three key sections. The first part of the paper will explain the research conducted so far. In the second part, the examination of the surface parameters that are necessary for us to obtain the connection between the additive material and the coating will be presented. Taking into consideration the first two units, the third unit will explain: the selection of the experiment plan, input factors, output factors as well as the method of conducting the experiment.
\end{abstract}

Keywords: additive technology; application of protective coatings; differential scanning calorimetry analysis; free surface energy and surface adhesion; polymer mold; thermogravimetric analysis (TGA)

\section{INTRODUCTION}

The world is entering the fourth industrial revolution and there are increasing demands in terms of the production process that needs to be more efficient [1-3]. Therefore, additive production sets as a logical choice in terms of better energy use in the production of complex products [4-6]. Through research and through practical work on devices for additive technology, shortcomings have been identified that will continue to represent an obstacle to the full implementation of additive technology in the production process. One of the major disadvantages of additive production is the material that is limited to a particular type of polymer developed exclusively for additive technology. Very little research has been conducted on the topic of making molds from polymer formations with additive technology. New markets require higher speed of delivery of the finished product as well as personalization according to the customer's wishes. The Croatian market usually has small series or serial production. Therefore, the cost of making a metal mold is quite expensive and time-consuming process. Previous research on molds made of polymer materials by additive technology goes in the direction of very small series of some 200 moldings. [7-9] and no emphasis is placed on making hundreds of products in this way. The life cycle of a polymer mold depends on the material to be injected into the mold. The number of molds can vary greatly from 10 to 100 [10]. Preliminary testing of coatings was conducted in Croatia and Bosnia and Hercegovina. Fig. 1 shows a mold made by additive technology and a mold obtained after injection molding. Polymer molds made by additive technology are produced in the world, but their life cycle is short and the cost-effectiveness of the same is questionable. As a part of the doctoral thesis, the field of coating polymer formations made by additive technology will be researched. One of the goals of the doctor thesis is to significantly extend the life cycle of polymer molds obtained by additive technology. At the same time, the impact on the environment will be reduced because it will not be necessary to use a large amount of energy for the particle separation treatment of the mold.

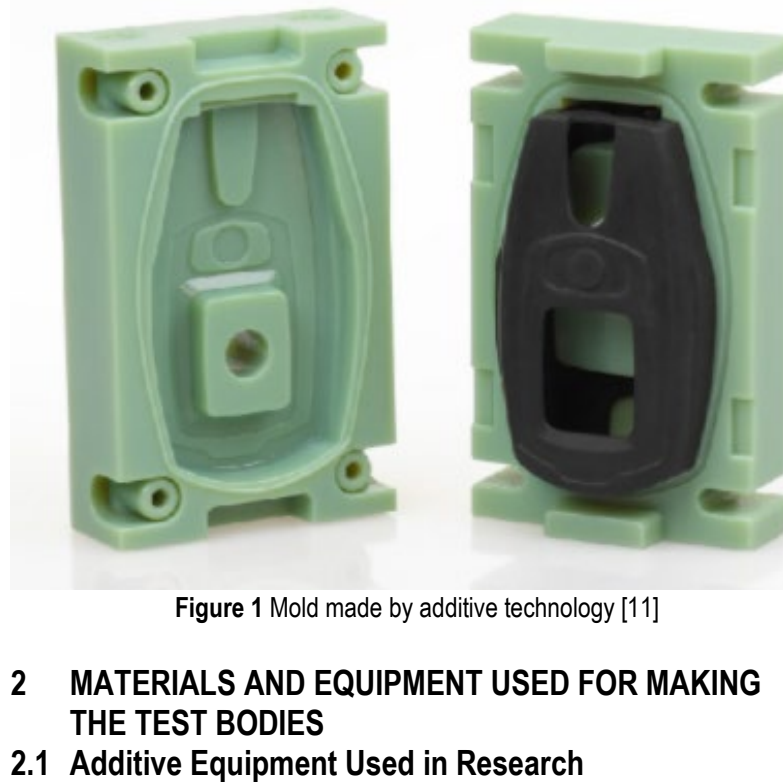

The test bodies were made in the laboratory for polymer processing of the Faculty of Mechanical Engineering and Naval Architecture in Zagreb. Two types of technologies were used to make the test bodies: sedimentation -FDM (Fig. 2) and Hybrid 3D printing and stereolithography (Fig. 3). The Makerbot additive device has two heads, ie nozzles, and can simultaneously make parts from two different materials or from the same material only different colors. It can also be completely closed, which prevents heat loss, which is suitable for 3D production of objects, and therefore it is extremely suitable for ABS polymer material. It can also work with PLA, PS$\mathrm{HI}$ and TPE materials. 


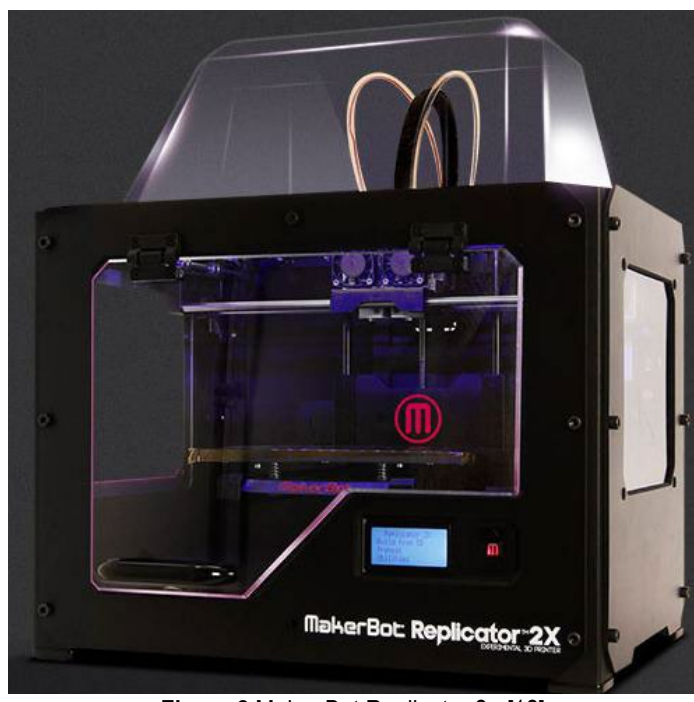

Figure 2 Maker Bot Replicator 2× [12]

Second device that was used to make the test bodies was the Stratasys 350 Connex. It is a device that allows the printing of several different types of materials at the same time, thus eliminating the need for subsequent joining of individual parts.

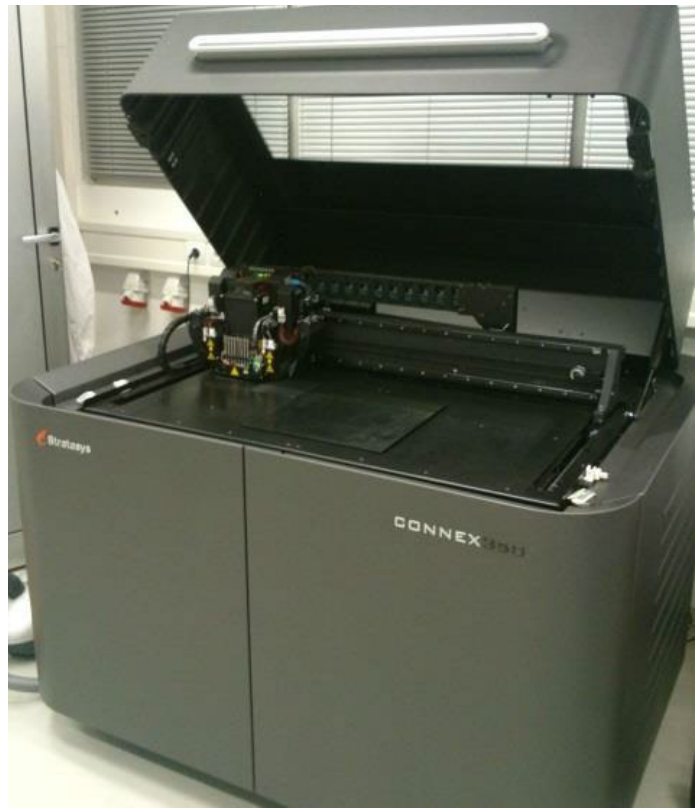

Figure 3 Objet Connex 350 at the Faculty of engeneering and Naval arhitecture in Zagreb

\subsection{Types of Material Used for the Production of Test Bodies}

The polymer material ABS (Acrylonitrile butadiene styrene) was used on the additive device Maker Bot Replicator $2 \times$. ABS is the most common material in FDM additive devices. In addition to the characteristics listed above, its price, suitability for use for various purposes, as well as simple finishing by grinding, in order to achieve smooth surfaces and remove serrated edges and painting, are also very important [13].

Fig. 4 shows test bodies made of ABS polymer using Maker Bot Replicator $2 \times$.

In the first phase, test bodies were made according to ISO EN ISO 527:2012 standard.

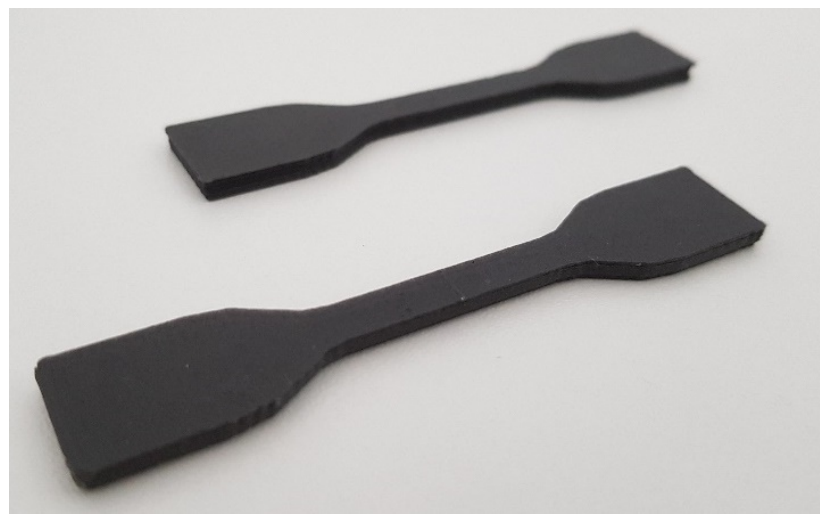

Figure 4 Test bodies made on FDM device

The additive device Objet Connex 350 offers a wide range of materials. Three types of materials were chosen: Digital ABS, Tango Black Plus and Vero Clear. Digital ABS Plus is a standard material that has approximately the same properties as the technical polymer ABS. The most common use is for parts that have high demands on mechanical properties as well as resistance to high temperatures. Tango and TangoPlus are similar in properties to elastomeric materials such as rubber and are ideal for testing and verifying visual, tactile and functional applications such as grips, seals and footwear. Vero Clear is similar to polymer - acrylic and, achieving 95\% light transmission, is useful for prototypes of glass, transparent polymers or transparent packaging [14]. Fig. 5 shows the test bodies after production on the additive device Stratasys Connex 350. Since the first phase examens different covers or protective coatings, it was decided to change the test body dimension from ISO EN ISO 527: 2012 to $24 \times 80 \times$ $10 \mathrm{~mm}$.

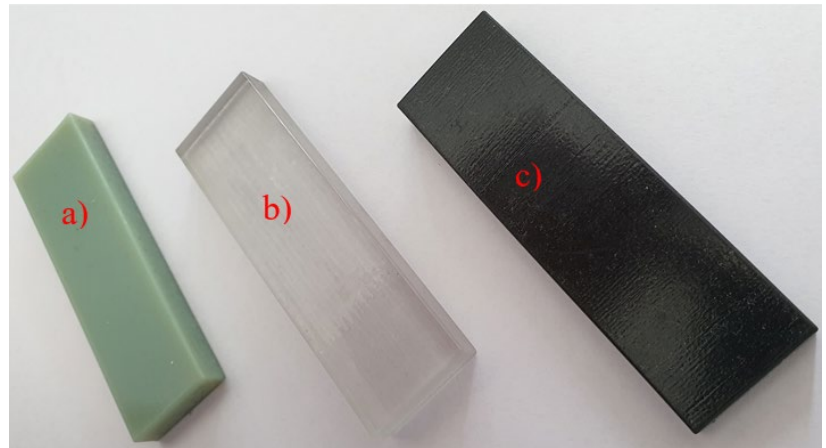

Figure 5 Test bodies a) Digital ABS b) Vero Clear c) Tango Black Plus

\subsection{Types of Procedures of Applying Protective Coatings}

Through previous research, which has been explained in earlier chapters, the problem of mold temperature has been identified, which significantly shortens its life cycle. The company Nova Chem from Karlovac provided us with two temperature coatings based on metal coatings. The first was based on Zinc and temperature resistance up to $400{ }^{\circ} \mathrm{C}$ (Novasil zinc 400) while the second was based on aluminum with temperature resistance up to $600{ }^{\circ} \mathrm{C}$ (Novasil al 600). The technologist explained that an acrylic coating must be used to apply both coatings. Fig. 6 shows the coating applied to the test body according to ISO EN ISO 527:2012 made of ABS material with additive technology FDM. Before coating, both test bodies were 
coated with acrylic coating for better adhesion of both coatings (Novasil zinc 400 and Novasil al 600).

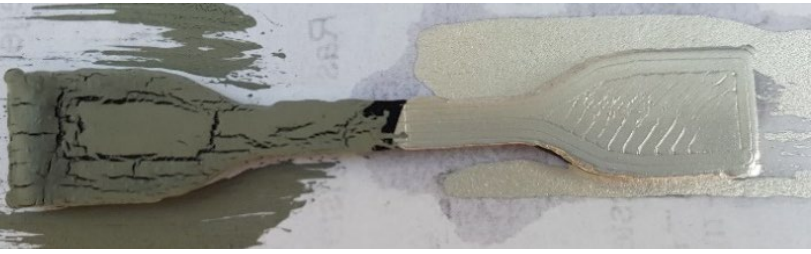

Figure 6 Absorption of Novasil cink 400 and Novasil al 600 coating

As seen in Fig. 6, Novasil zinc 400 (left side) was not well absorbed into the surface. Novasil al 600 was well absorbed into the surface thus research was continued with this coating. The coated test bodies were then subjected to a temperature of $180{ }^{\circ} \mathrm{C}$ for 10 minutes. The test bodies began to deform in all directions but the coating remained stable and did not begin to separate from the surface. (Fig. 7)

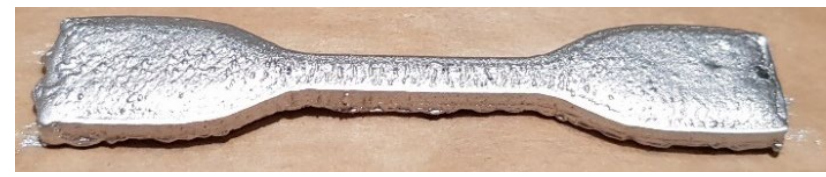

Figure 7 Test body protected with Novasil al 600 after heating on $180^{\circ} \mathrm{C}$

Another way of applying the metal coating was by vacuum metallization in the KOOGLE trade by evaporating aluminum on a test body which is done for decorative purposes (Fig. 8).

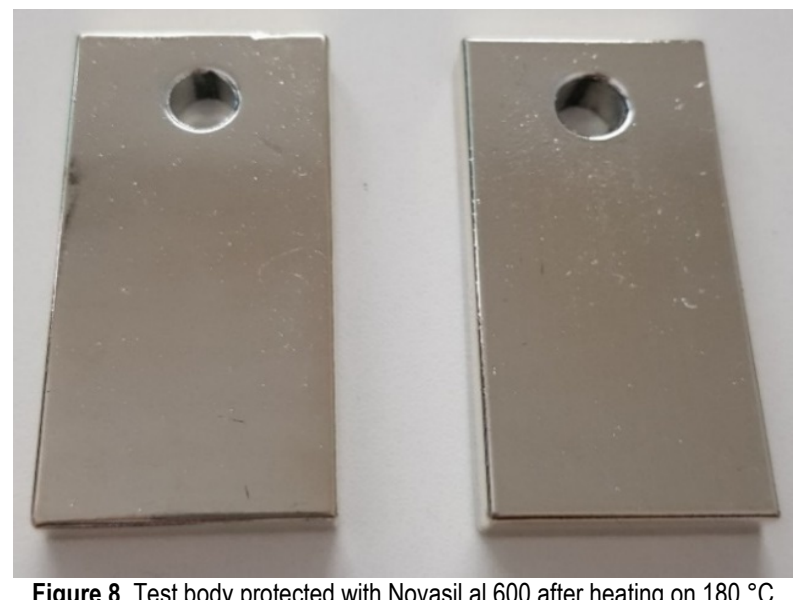

Aluminum metallization was performed on test bodies made on the additive device Stratasys Connex 350, and the test body material was Digital ABS. Two holes were made on the top for easier acceptance of the test bodies. Since the polymer is metallized using aluminum for decorative purposes, it is necessary to apply a colorless varnish after each procedure. In the company Tiplon d.o.o. electroplating of copper was performed on all three materials of the test bodies. The company has three electroplatination systems CHEMCUT, ATOTECH and LANTRONIC. Since the company is engaged in the production of printed electronic boards, all systems are exclusively meant for this process. Fig. 9 shows copper layering, ie. electroplatinization of test bodies made of a) Digital ABS b) Vero Clear c) Tango Black Plus.

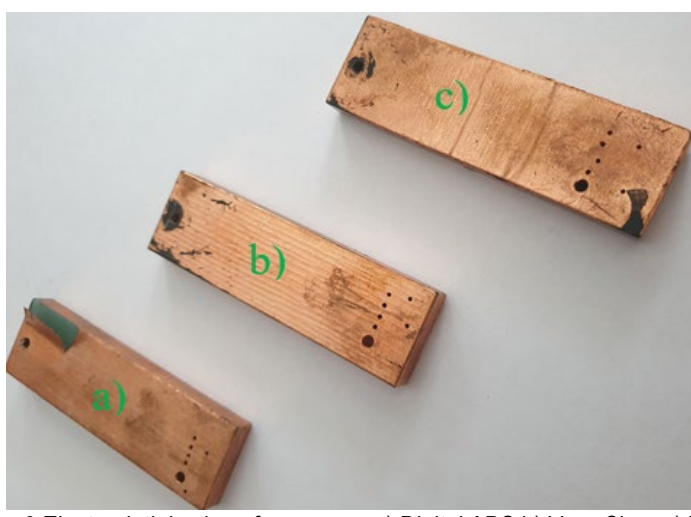

Figure 9 Electroplatinization of copper on a) Digital ABS b) Vero Clear c) Tango Black Plus.

The copper coating was not well absorbed into the surface on any of the three test bodies. A copper sheath was made and it was not sufficient to apply some other material by electroplatination. For this purpose, the application of an electrically conductive primer, that is usually applied in the automotive industry during ecstrostatic painting, was chosen. The company AD Plastik met the needs and applied a layer of electrically conductive primer to three test bodies. In Tiplon, a copper layer was applied again using electroplatination. Fig. 10 shows the three test bodies. Two phases of copper coating application were performed: the first phase is the installation of an electrically conductive primer for electrostatic paint application (Fig. 10a)) while the second phase is the application of copper. Fig. 10b) shows a directly connected test body to the electrode. Due to the need for strong current, electroplatination did not occur. Fig. 10c) shows the subsequent application of an electrically conductive paste after which a labile copper layer was obtained.

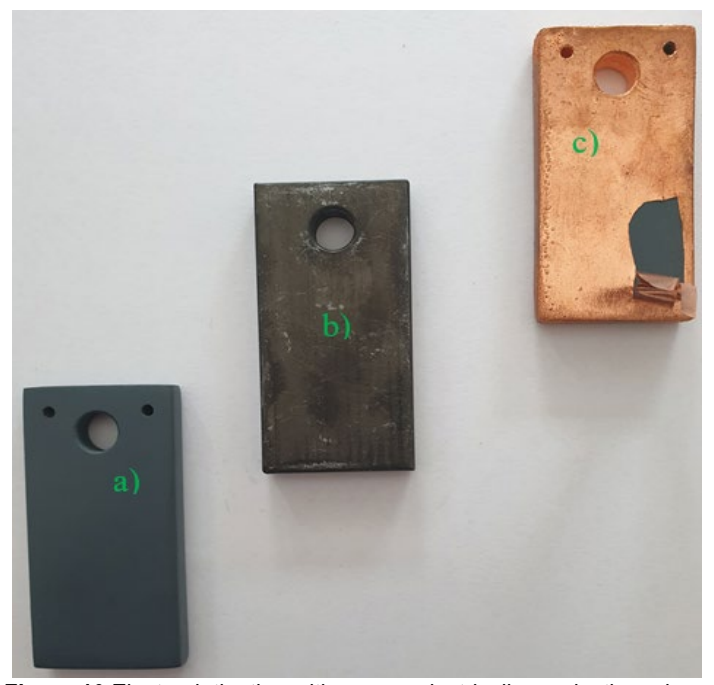

Figure 10 Electroplatination with copper-electrically conductive primer

The next test was performed at the Faculty of Mechanical Engineering and Naval Architecture in Zagreb at the Department of Materials. The nano layer was applied using sol-gel technology. The sol-gel process is an innovative low-temperature method of preparing homogeneous glasses and ceramic materials from the liquid phase. The main advantages of this method are low process temperature, excellent control of purity and stoichiometry of starting materials, possible preparation of 
porous and non-porous homogeneous oxide films, their easy application on large surfaces and good control of film thickness. In addition, it is a cheap and environmentally friendly technique because, as already mentioned, it represents a potential replacement for toxic chromium coatings. A significant feature of the sol-gel process is, that by simply changing the process parameters $(\mathrm{pH}$, temperature, type of solvent, type of catalyst, density and viscosity of the initial solution) the desired properties of the final material such as chemical and corrosion resistance, hardness, density, porosity, optical transparency, thermal resistance and electrical properties can be achieved. The flexibility and simplicity of sol-gel technology have made it one of the leading procedures in the preparation of new materials of different composition, structure, functionality and morphology [15].

Fig. 11 shows a ceramic layer applied using sol gel technology. Sol gel technology is suitable because the layer thickness is in nanometers, which does not significantly affect our dimensions. The layer is almost invisible except on a test body made of Digital ABS which shows a yellow trace of sol gel technology.

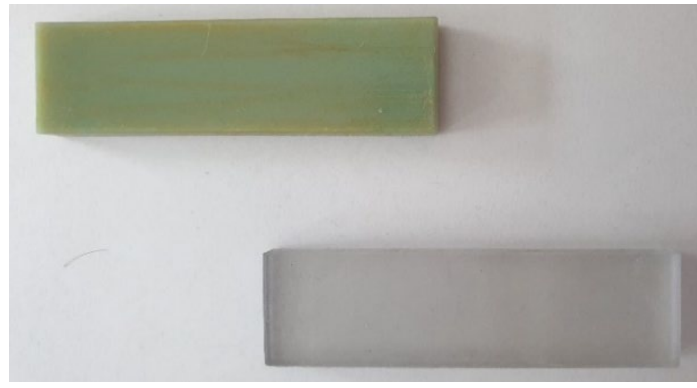

Figure 11 Layer appliying using sol gel technology

The final test of the application of protective coatings was performed with a coating based on Loctite SF 7900 Ceramic. Firm Raos 1.1.c. provided us with Loctite SF 7900 Ceramic protective spray, which is used in the field of welding as protection of welding nozzles from "slag".

Fig. 12 shows the application of protective ceramic coating using a spray Loctite SF 7900 Ceramic. Protective layer is easily absorbed on the surface of the test body. The test body has a significantly finer surface structure.

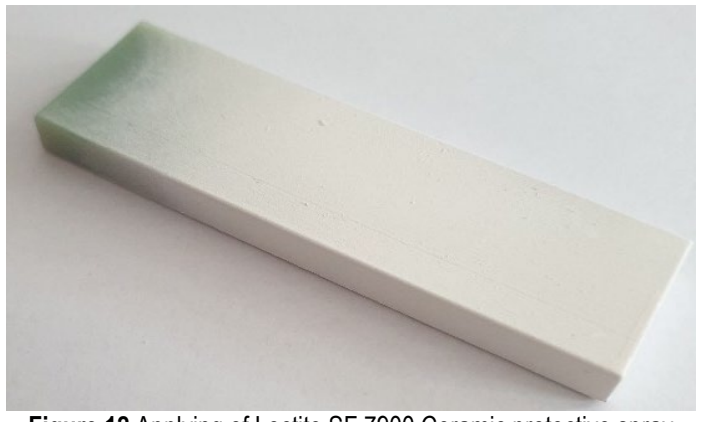

Figure 12 Applying of Loctite SF 7900 Ceramic protective spray

\subsection{Research Results}

After conducting research in Croatia, the conclusion is that, unfortunately, Croatian industry has little capacity to develop new technologies. Fig. 13 shows all the test bodies on which the research was conducted.

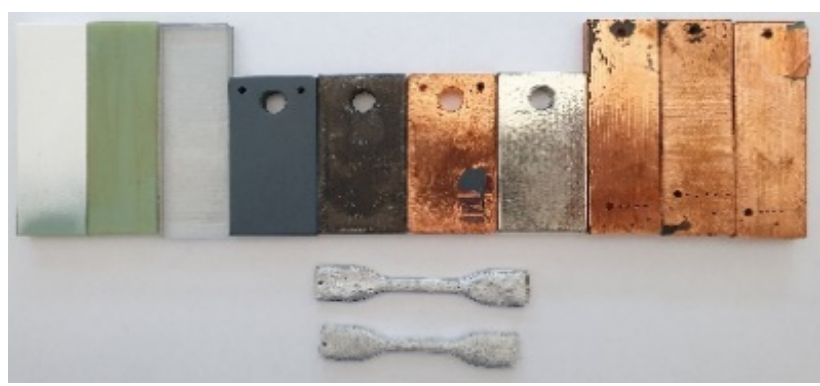

Figure 13 Test bodies after examination

During first tests, it was recognized that it is possible to install a protective layer that provides protection against elevated temperatures. Novasil al 600 was proven as a good choice for protection and adhesion to the test surface. The application of the aluminum layer by metallization proved to be a good technology for the absorption of aluminum into the surface of the test body. The problem occurs because the aluminum coating itself is soft and unsuitable for applying some other coatings due to surface oxidation. The application of a copper coating shows the need for a quality electrically conductive primer. In the world there are electrically conductive primers based on silver and zinc. Copper is suitable for applying almost any metal layer by electroplatination, which seems to be the best option for further research. Applying ceramics using sol gel technology is currently not an interesting procedure given the impossibility of quality research in Croatian laboratories. Applying a ceramic coating using a spray seems to be a simple process that will be explored in regard to surface exploitation and wear. Conclusion of research conducted so far:

- It is necessary to define surface parameters (adhesion, free surface energy, etc.).

- Based on the surface parameters, make a layer that will be the link between the additive material and the protective coating.

- Optimize and standardize the process for all other types of additive materials.

\section{EXAMINATION OF SURFACE PARAMETERS}

Tab. 1 shows the selected samples for further research of surface parameters and their labeling.

Table 1 List of samples and their labeling
\begin{tabular}{|l|c|}
\hline Sample & Label \\
\hline Digital ABS & ABS \\
\hline Digital ABS + copper & $\mathrm{Cu}$ \\
\hline Digital ABS + electrically conductive primer & coating \\
\hline
\end{tabular}

\subsection{Thermal Surface Analysis 3.1.1 Thermogravimetric Analysis (TGA)}

The thermal stability and the proportion of nondegradable residue of the Digital ABS polymer sample was determined by thermogravimetric analysis (TGA) on the instrument TA Instruments Q500. Measurements were performed in the temperature range from 25 to $900{ }^{\circ} \mathrm{C}$ at a heating rate of $10^{\circ} \mathrm{C} \mathrm{min}^{-1}$, in an inert nitrogen atmosphere 
with a flow rate of $60 \mathrm{~cm}^{3} \mathrm{~min}^{-1}$. The mass of the sample was about $10 \mathrm{mg}$.

\subsubsection{Differential Scanning Calorimetry (DSC)}

The differential thermal properties of the Digital ABS polymer sample were determined on a DCS $823^{\mathrm{e}}$ manufacturer Mettler Toledo. The measurement was performed in an aluminum bowl with a lid with a volume of $40 \mu \mathrm{L}$ manufactured by Netch. The instrument was calibrated with indium and zinc according to the standard procedure which is an integral part of the computer program of the DSC instrument. The weight of the sample was about $10 \mathrm{mg}$. DSC analysis was performed by the procedure in two heating cycles and a cooling cycle in the temperature range from $-85^{\circ} \mathrm{C}$ do $300{ }^{\circ} \mathrm{C}$ at a rate of 10 ${ }^{\circ} \mathrm{C} \mathrm{min}^{-1}$ in an inert nitrogen atmosphere with a flow rate of $60 \mathrm{~cm}^{3} \mathrm{~min}^{-1}$. The first heating cycle was performed to undo the thermal history of sample preparation. The thermogram of the second heating cycle was analyzed.

\subsubsection{Determining of Free Surface Energy}

The free surface energy of the following samples: Digital ABS, copper $(\mathrm{Cu})$ and coating sample was determined by the contact angle measurement technique on the instrument DataPhysics OCA 20 Instruments GmbH. Test liquids, water, formamide and diiodomethane, of known values of free surface energy, were used to determine the contact angle (Tab. 2).

Table 2 Components of free surface energy of test liquids $[16,17]$

\begin{tabular}{|l|c|c|c|}
\hline \multirow{2}{*}{ Test liquid } & \multicolumn{3}{|c|}{ Free surface energy / $\mathrm{mJ} \mathrm{m}^{-2}$} \\
\cline { 2 - 4 } & $\gamma$ & $\gamma^{d}$ & $\gamma^{p}$ \\
\hline Water & 72,8 & 21,8 & 51,0 \\
\hline Formamid & 58,0 & 39,0 & 19,0 \\
\hline Dijodometan & 50,8 & 50,8 & 0,0 \\
\hline
\end{tabular}

Volume of the drops of test liquids was $2 \mu \mathrm{L}$. The mean value of the contact angle for each test liquid was obtained based on the measurement of 10 - 15 drops. Deviations of the results from the mean value were $\pm 3^{\circ}$. The free surface energy of the tested samples was calculated using the computer program SCA 20 (Version 2.01, Data Physics Instruments, GmbH, 2001), using twoparameter models, Owens Wendt (Eq. (1))[16] and Wu (Eq. (2))[17].

$$
\begin{aligned}
& \gamma_{l v}(1+\cos \theta)=\sqrt{\gamma_{l}^{d} \gamma_{s}^{d}}+\sqrt{\gamma_{l}^{p} \gamma_{s}^{p}} \\
& \gamma_{l v}(1+\cos \theta)=\frac{4 \gamma_{l}^{d} \gamma_{s}^{d}}{\gamma_{l}^{d}+\gamma_{s}^{d}}+\frac{4 \gamma_{l}^{p} \gamma_{s}^{p}}{\gamma_{l}^{p}+\gamma_{s}^{p}}
\end{aligned}
$$

where $\gamma_{l v}$ is the total free surface energy of test liquid, $\gamma_{l}^{d}$ and $\gamma_{l}^{p}$ are the dispersed and polar component of the surface energy of the test liquid, $\gamma_{s}^{d}$ and $\gamma_{s}^{p}$ are the dispersed and polar component of the surface energy of the test sample, and $\theta$ is the contact angle.

\subsubsection{Results and discussion}

a) Results of TGA analysis:

Thermogravimetric analysis examined the thermal stability and the proportion of non-degradable residue of Digital ABS polymer (ABS). The thermal stability of the material was estimated based on the characteristics obtained from the TG and DTG curves; $T_{\text {onset } 1}$ initial temperature of the $1 \mathrm{st}$ decomposition stage, $T_{\text {end set } 1}$ end temperature of the $1 \mathrm{st}$ decomposition stage, $T_{\text {onset } 2}$ initial temperature of the $2 \mathrm{nd}$ decomposition stage, $T_{\text {end set } 2}$ end temperature of the 2nd decomposition stage, $T_{\max 1}$ maximum decomposition temperature of the $1 \mathrm{st}$ decomposition stage, $T_{\max 2}$ temperature of maximum speed of the 2nd stage of decomposition, residue - proportion of non-degradable residue. The TG and DTG curves of the Digital ABS sample are shown in Fig. 14, and the readings are given in Tab. 3 .

\begin{tabular}{|c|c|c|c|c|c|c|c|c|c|}
\hline \multirow{2}{*}{ Sample } & \multicolumn{9}{|c|}{ Table 3 Results of thermogravimetrc analysis } \\
\cline { 2 - 12 } & $T_{\text {onset } 1} /{ }^{\circ} \mathrm{C}$ & $T_{\text {end set } 1} /{ }^{\circ} \mathrm{C}$ & $T_{\text {onser } 2} /{ }^{\circ} \mathrm{C}$ & $\mathrm{T}_{\text {end set2 }} /{ }^{\circ} \mathrm{C}$ & Residual $/ \%$ & $\Delta m_{1} / \%$ & $\Delta m_{2} / \%$ & $T_{\max 1} /{ }^{\circ} \mathrm{C}$ & $T_{\max 2} /{ }^{\circ} \mathrm{C}$ \\
\hline \multirow{2}{*}{ Digital ABS } & 314,5 & 397,8 & 397,8 & 461,5 & 1,996 & 22,5 & 75,5 & 327,0 & 450,4 \\
\hline
\end{tabular}

In the range of lower temperatures from 25 to about $300{ }^{\circ} \mathrm{C}$ where low volatile matter and water evaporate there is no significant weight loss and the sample loses about $1.3 \%$ of the initial mass. Further heating above $300{ }^{\circ} \mathrm{C}$ starts the thermal decomposition of ABS in two stages of decomposition. Acrylonitrile butadiene styrene (ABS) is an amorphous thermoplastic therpolymer consisting of a styrene-acrylonitrile copolymer (SAN) as a continuous phase in which the finely dispersed rubber phase is poly (butadiene) (BR). [18] The first stage of degradation corresponding to the degradation of polybutadiene [19-21] starts at $314.5^{\circ} \mathrm{C}$ and ends at $397.8^{\circ} \mathrm{C}\left(\Delta T=83.3{ }^{\circ} \mathrm{C}\right)$ during which the polymer loses $22.5 \%$ weight of the initial mass. The second stage of degradation reflecting the degradation of SAN copolymers [19-21] starts at $397.8^{\circ} \mathrm{C}$ and ends at $461.5^{\circ} \mathrm{C}\left(\Delta T=63.7^{\circ} \mathrm{C}\right)$ during which the polymer loses $75.5 \%$ weight of the initial mass. The DTG curve shows two maxima at about $330^{\circ} \mathrm{C}$ and $450{ }^{\circ} \mathrm{C}$, Tab. 3 , which correspond to the maximum decomposition rates of polybutadiene and SAN copolymers. Analysis of the DTG maximum shows that the rate of degradation of the polybutadiene phase is higher than the degradation of SAN copolymers. A closer study of the first DTG maximum shows that it contains two overlapping maxima $\left(T_{\max 1}=\right.$ $327,0^{\circ} \mathrm{C}$ i $335,2^{\circ} \mathrm{C}$ ), which indicates the complexity of the degradation reaction of ABS polymer. After $900{ }^{\circ} \mathrm{C}$, about $2 \%$ of the non-degradable residue remains.Thermal decomposition of ABS takes place by a radical process in which end-chain cleavage and statistical cleavage of polymer chains occur, and butadiene-rich domains are cited as the most sensitive degradation sites. Literature mentiones [22] acetophenone, acrylonitrile, benzaldehyde, 
cresol, dimethylbenzene, hydrogen cyanide, methyl styrene, styrene as the main products of thermal decomposition.

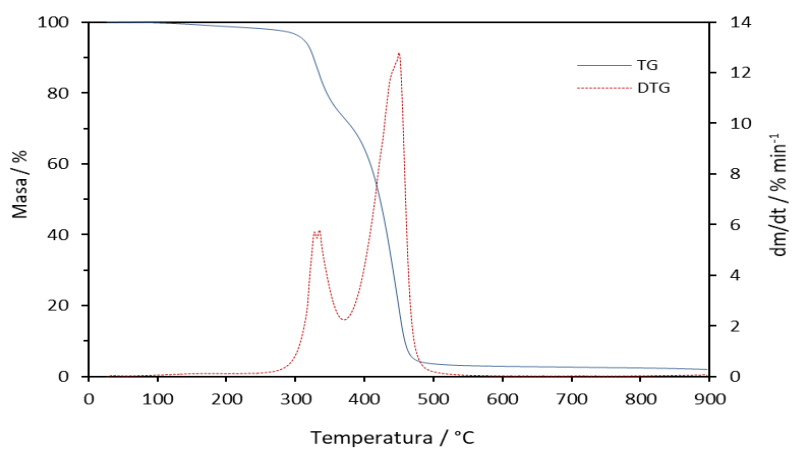

Figure 14 TG i DTG curves of Digital ABS sample

b) Results of the DSC analysis:

The thermal properties of the sample were examined by DSC analysis. The normalized DSC curve of the Digital ABS sample of the second heating cycle is shown in Fig. 15 and the glass transition temperatures read from the thermogram are given in Tab. 4.

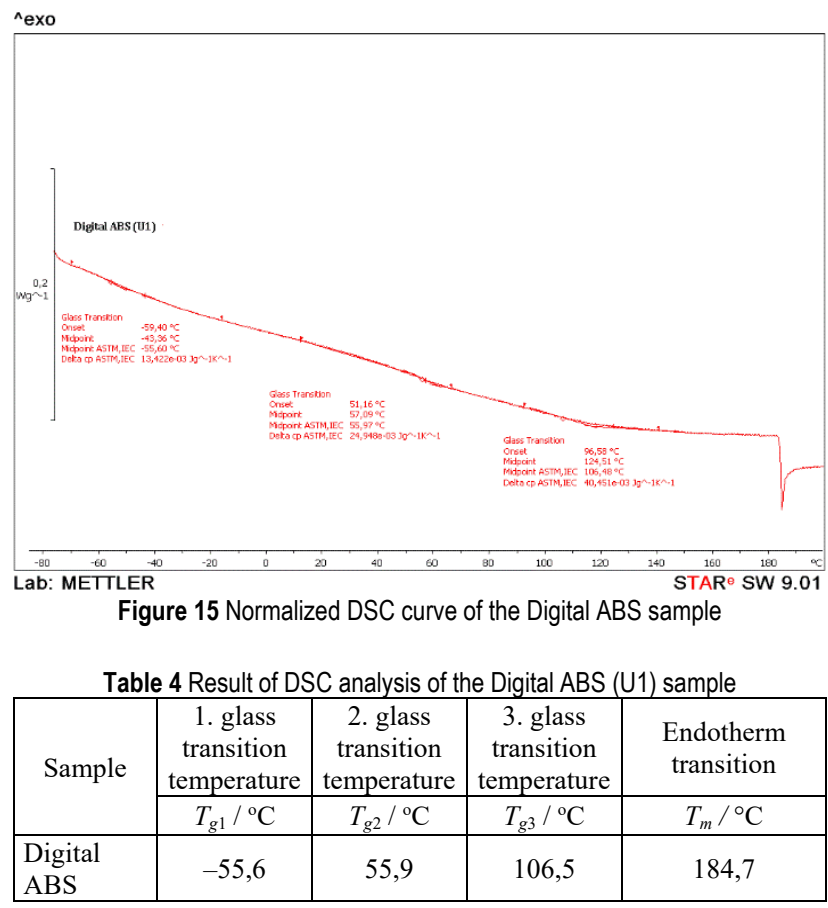

In the first and second heating cycle of the Digital ABS sample, three glass transition temperaturares visible on the DSC curve, ie the temperature ranges that define the transition from the vitreous to the viscoelastic state. The results indicate the amorphous structure of Digital ABS polymers and the presence of separated phases in $\mathrm{ABS}$ polymer. The first vitreous transition at $-48.4{ }^{\circ} \mathrm{C}$ can be attributed to the polybutadiene phase $(\mathrm{PB})$ [23] while the third vitreous transition at $105.5{ }^{\circ} \mathrm{C}$ can be attributed to polyacrylonitrile (AN) [21]. The absence of a single vitreous transition of ABS polymer indicates the immiscibility of the SAN and PB phases.

As ABS is amorphous, it has no defined melting temperature. The vitreous transition at $56{ }^{\circ} \mathrm{C}\left(T_{g 2}\right)$ and the endothermic transition $\left(T_{m}\right)$ around $185{ }^{\circ} \mathrm{C}$ represent the melting of the material used to mix the SAN and butadiene copolymer. Reed et al. [24, 25] confirmed that the addition of mold lubricant during ABS production remains within the ABS matrix. Namely, manufacturers often use some unknown chemicals to reduce the viscosity and narrow the extrusion/compression parameters. Therefore, the vitreous transition temperature $\left(T_{g 2}\right)$ at $56{ }^{\circ} \mathrm{C}$ and the melting temperature $\left(T_{m}\right)$ at $185^{\circ} \mathrm{C}$ can be attributed to the melting of the materials used during the production of ABS.

\subsection{Surface Parameters 3.2.1 Free Surface Energy}

Surface characterization of ABS, copper $(\mathrm{Cu})$ and coating samples was performed using the contact angle technique with liquids of known free surface energy values. The experimentally obtained values of the contact angles are shown in the table.

\begin{tabular}{|l|c|c|c|}
\multicolumn{4}{c}{ Table5 Values of the contact angle of the samples } \\
\hline \multirow{2}{*}{ Sample } & \multicolumn{3}{|c|}{ Contact angle $\theta /{ }^{\circ}$} \\
\cline { 2 - 4 } & Water & Formamid & Diiodomethane \\
\hline ABS & $60,1 \pm 0,0$ & $64,9 \pm 2,2$ & $47,1 \pm 1,4$ \\
\hline $\mathrm{Cu}$ & $49,2 \pm 2,6$ & $40,6 \pm 1,0$ & $42,2 \pm 3,9$ \\
\hline coating & $65,8 \pm 1,7$ & $67,6 \pm 1,1$ & $55,4 \pm 1,8$ \\
\hline
\end{tabular}

A higher value of the contact angle with water on the ABS and coating samples indicates the hydrophobic nature of the surface, while a lower value for the copper $(\mathrm{Cu})$ sample indicates the hydrophilic nature of the surface. The obtained values of the contact angles were used to calculate the free surface energy using the geometric mean model (Owens Wendt) (Eq. (1)) and the harmonic mean model (Wu) (Eq. (2)).

The values of the disperse and polar components and the values of the total free surface energy of the tested samples are given in Tab. 6 .

Table 6 Values of the free surface energies calculated using Owens Wendt and Wu model

\begin{tabular}{|l|c|c|c|c|c|c|}
\hline \multirow{2}{*}{ Sample } & \multicolumn{3}{|c|}{ Owens Wendt $/ \gamma / \mathrm{mJ} \mathrm{m}^{-2}$} & \multicolumn{3}{|c|}{$\mathrm{Wu} / \gamma / \mathrm{mJ} \mathrm{m}^{-2}$} \\
\cline { 2 - 7 } & $\gamma_{\mathrm{s}}^{\mathrm{d}}$ & $\gamma_{\mathrm{s}}^{\mathrm{p}}$ & $\gamma_{\mathrm{s}}$ & $\gamma_{\mathrm{s}}^{\mathrm{d}}$ & $\gamma_{\mathrm{s}}^{\mathrm{p}}$ & $\gamma_{\mathrm{s}}$ \\
\hline $\mathrm{ABS}$ & 30,2 & 8,4 & 38,6 & 31,2 & 11,1 & 42,3 \\
\hline $\mathrm{Cu}$ & 33,8 & 19,5 & 53,3 & 33,9 & 22,4 & 56,3 \\
\hline coating & 26,2 & 9,4 & 35,6 & 27,8 & 11,8 & 39,6 \\
\hline & \multicolumn{3}{c}{${ }_{\mathrm{s}}^{\mathrm{d}}-$ disperse component, $\gamma_{\mathrm{s}}^{\mathrm{p}}$ - polar component, $\gamma_{\mathrm{s}}$ - total free surface } \\
energy
\end{tabular}

Free surface energy data provide important information about surface properties of materials and allow monitoring of properties during the preparation of multiphase systems and changes in material properties during aging.

A higher value of the total surface energy of copper $(\mathrm{Cu})$ indicates a higher surface activity compared to the ABS and coating samples (Tab. 6).

Polymeric materials are characterized by a low value of free surface energy which is a consequence of the chemical structure of the polymer, i.e. the presence of nonpolar groups in the polymer chain. Similar values of the dispersion components of the surface of the ABS, $\mathrm{Cu}$ and coating samples indicate the equal possibility of the 
contribution of the dispersion forces of all tested materials. A significant difference is visible in the polar, $\gamma^{p}$ component. A higher value of the polar component, $\gamma^{p}, \mathrm{Cu}$ indicates a more polar nature of the metal surface and it can be expected that $\mathrm{Cu}$ will have a greater ability to establish polar interactions, which may result in better adhesion to polar substances. On the other hand, the lower value of the polar component of ABS and coating samples indicates their non-polar nature and the possibility of establishing weaker interactions with polar substances.

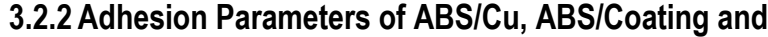 Coating/Cu}

Surface properties are defined by the nature ie chemical structure of the surface of the material on which the adhesion properties depend. Most polymeric materials have a non-porous and chemically inert surface, non-polar or hydrophobic in nature, and low surface energy. This is the reason that the surfaces of polymeric materials show a weak tendency to wetting, ie the impossibility of achieving good adhesion with high surface energy materials such as metals, coatings, varnishes and so on. A prerequisite for strong (optimal) adhesion between the polymer and the coating is good wetting, which ultimately results in good usable properties of the material. Adhesion is affected not only by the nature and properties of the surface but also by impurities that represent a weakly binding layer on the surface of the material.

One way to estimate the adhesion on the polymer/coating interface or polymer/metal is to calculate the adhesion parameters of binary systems: thermodynamic adhesion work $\left(W_{12}\right)$, free interfacial energy $\left(\gamma_{12}\right)$ and spread coefficient $\left(S_{12}\right)$. The presence of interactions at the interface of the two phases, although important, is only one of the factors influencing the properties of multiphase systems.

For polymer/coating component pairs, the adhesion parameters can be calculated using the values of free surface energies of the two components that make up the interface, polymer 1 and coating 2 according to the following expressions:

$W_{12}=\gamma_{1}+\gamma_{2}-\gamma_{12}$

$\gamma_{12}=\gamma_{1}+\gamma_{2}-4 \frac{\gamma_{1}^{d} \gamma_{2}^{d}}{\gamma_{1}^{d}+\gamma_{2}^{d}}-4 \frac{\gamma_{1}^{p} \gamma_{2}^{p}}{\gamma_{1}^{p}+\gamma_{2}^{p}}$

$S_{12}=\gamma_{1}-\gamma_{2}-\gamma_{12}$

According to the literature, as conditions regarding optimal adhesion are stated the maximum thermodynamic work of adhesion $\left(W_{12}=\max \right)$, the positive value of the spread coefficient $\left(S_{12} \geq 0\right)$ and the minimum value of free interfacial energy $\left(\gamma_{12}\right)$ [26].

Tab. 7 shows the values of the adhesion parameters of the $\mathrm{ABS} / \mathrm{Cu}, \mathrm{ABS} /$ coating and coating/ $\mathrm{Cu}$ pairs obtained according to Eq. (3), Eq. (4) and Eq. (5).
Table 7 Parameters of the adhesion of the $A B S / C u, A B S /$ coating and caoting/Cu pairs

\begin{tabular}{|l|c|c|c|}
\hline \multirow{2}{*}{ Pairs } & \multicolumn{3}{|c|}{ Parameters of adhesion $/ \mathrm{mJ} \mathrm{m}^{-2}$} \\
\cline { 2 - 4 } & $\gamma_{12}$ & $W_{12}$ & $S_{12}$ \\
\hline ABS/Cu & 3,92 & 94,68 & $-17,92$ \\
\hline ABS/coating & 0,22 & 81,68 & 2,48 \\
\hline coating/Cu & 3,89 & 92,01 & $-20,59$ \\
\hline
\end{tabular}

According to the obtained results, it can be seen that the pair $\mathrm{ABS} / \mathrm{Cu}$ and coating/Cu do not meet the conditions for optimal adhesion, while the pair ABS / coating satisfies all three conditions for optimal adhesion. The values of the thermodynamic work of adhesion indicate the presence of certain interactions on the interface of $\mathrm{ABS} / \mathrm{Cu}$, $\mathrm{ABS} /$ coating and coating/Cu.

The higher value of the interfacial energy $\gamma_{12}$ and the negative value of the spread coefficient, $S_{12}$ para $\mathrm{ABS} / \mathrm{Cu}$ and coating $/ \mathrm{Cu}$, Tab. 7 , indicate poor adhesion and the possibility of separation of copper from the surface of ABS and coating.

The minimum value of the interfacial energy $\gamma_{12}$ and the positive value of the spread coefficient, $S_{12}$ of the ABS/coating pair indicate a strong (optimal) adhesion between the ABS and the coating.

\subsection{Conclusion of the Examination of Surface Parameters}

Since the area of coating polymer products obtained by additive technology was unexplored, it was necessary to examine the possibility of creating an intermediate layer that would be a link between the polymer formation and the coating.

Through surface tests, an image and a substrate were created for the development of an intermediate layer that will have a high degree of adhesion to the surface of the polymer formation, but also connect the protective coating with the polymer formation.

\section{PLANNING OF THE EXPERIMENT}

In further research, one of the objectives is to examine the effect of influential process parameters of injection molding in a hybrid mold (mold made by polymer-based additive technology coated with a protective coating) on the number of moldings. As it is important to obtain quality moldings, it is necessary to determine the optimal parameters for the production of a hybrid mold, which will increase the number of obtained moldings compared to previous research.

A central composite experimental plan was selected for further study. On the basis of polynomials of the second degree, the plan allows the display of the response surface that is investigated within the given limits. In this way, it will be possible to select the optimal parameters for making a hybrid mold regarding to the number of moldings.

\subsection{Central Composite Experiment Plan}

The central composite experimental plan belongs to the group of higher-order experimental plans, the so-called response surface method. The response surface method encompasses a set of statistical and mathematical methods that are applied to develop, improve, and optimize 
processes. Measurable size of product or process quality is called the response. The central composite plan of the experiment is a I .model $\left(2^{k}\right)$ extended by additional points (experimental states) in the center and points in the axes in order to allow the estimation of the parameters of the model of the II. order. The central composite plan of the experiment consists of $2^{k}$ states in the peaks (factor states), $2 k$ states in the axes and states in the center $(k-$ number of factors). The central composite experimental plan is an alternative to the $3 k$ model when compiling experimental model of the II. order because the number of performances is reduced compared to the complete factor model of the experiment. For $k=3$ (factors are $x_{1}, x_{2}$ and $x_{3}$ ), Fig. 16 shows a model of a central composite experiment that requires 15 experimental states $\left(2^{3}+2 \cdot 3+1\right)$. In the case of a complete factor experiment, 27 experimental states would be required [27].

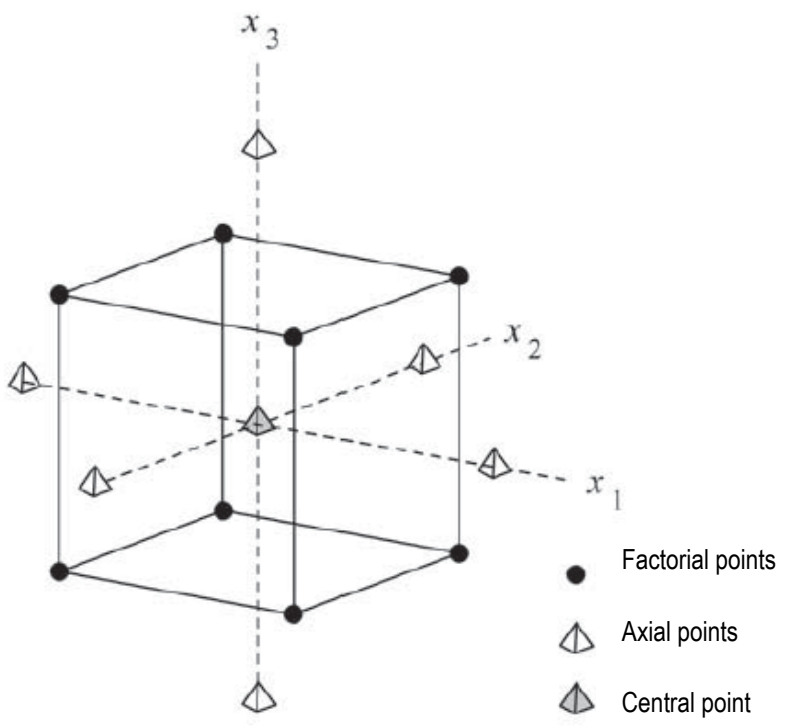

Figure 16 Central composite experiment plan regarding three factors [28]

A desirable feature of each experiment is the mutual independence of the assessments of the main effects and their interactions, which is achieved by the orthogonality and rotatability of the experiment. An experiment is orthogonal if the sum of the products of the coded states of any two columns in the experiment matrix is equal to zero.

The rotatability of the centrally composite experimental plan is achieved by adding the experimental state so that all states are equally distant from the center of the experiment, ie the rotatability depends on the so-called axial distance $\alpha$ (distance of the state in the axes from the center) [27].

The experiment is rotatabile if:

$$
\alpha=\sqrt[4]{f}
$$

where $F$ is a number of factor states $\left(F=2^{k}\right.$ in the case of a complete factorial experiment). in case of complete factor experiment). According to Eq. (6), in a case of two factors $\alpha=2^{2 / 4}=1,414$, and in the case of three factors $\alpha=2^{3 / 4}=$ 1,682 .

Additional states at the center of the experiment assist in order to be able to compare the measurement values of the dependent variable at the center of the experiment with the arithmetic mean for the rest of the experiment. If the arithmetic mean of the experimental center is significantly different from the total arithmetic mean of all other experimental states, then it can be concluded that the relationship between the experimental factor and the dependent variable is not linear. If the experiment is at least partially repeated, then the error of the experiment can be estimated from the variability of the repeated states. Because these states are performed under identical conditions, ie identical factor levels, the estimation of the experimental error from these data is independent regardless of the experimental model (linear or nonlinear) and regardless to the contains (higher-order interactions). Thus, the estimated experimental error is pure error, ie it is a consequence only of the uncertainty of measuring the dependent variable. The equation (II degree polynome) describing the process (response function) for the general case reads ( $\mathrm{k}$ experiment factor):

$$
\begin{aligned}
& \bar{y}=b_{0}+b_{1} x_{1}+\ldots+b_{k} x_{k}+b_{12} x_{1} x_{2}+b_{13} x_{1} x_{3}+\ldots+ \\
& +b_{k-1} x_{k-1} x_{k}+b_{11} x_{1}+b_{k k} x_{k}^{2}
\end{aligned}
$$

where the coefficients $b_{0}, \ldots, b_{k}$ are determined by applying the method of the minimum sum of square deviations of the calculated from the actual values [27].

\subsection{Determining the Parameters of the Experiment for Further Research}

As the first step, the optimization of the parameters of obtaining the interlayer between the additive formation and the protective coating is determined. The intermediate layer will be optimized in order to reduce the costs of applying the protective coating and to determine the input parameter, ie. by how much the geometry of the mold needs to be increased to obtain a mold of the desired dimension.

According to preliminary tests, the following are determined as input quantities for the implementation of the experiment plan:

- type of coating

- coating thickness

Given the available resources, the base polymer mold will be coated with a minimum of two different coatings.

The thickness of the coating will depend on the quality of the coating device, and at least two thicknesses of the coating will be made.

The outputs from the process to be studied will be:

- change in wall thickness in a certain cycle interval with respect to the original value. Before the first injection molding of the polymer into the mold, the wall thickness will be measured and each subsequent measurement will be compared with the initial size.

- The castability of the material that is injected into the mold. A mold that satisfies the standard casting test (test list, Archimedean spiral, etc.) will be made.

- Surface roughness in a particular polymer injection molding cycle.

Since our goal is to ensure the same conditions during injection molding, the making of the mold will be performed in the following ways: 
- $\quad$ one control nest without coating;

- two nests with one type of coating and one thickness and

- two nests with a different type of coating and one thickness.

In the described way, possible variations in the parameters of the machine and the material that is injected, which could have an impact on the result of the output values, will be minimized.

\section{CONCLUSION}

One of the requirements that is being imposed to modern processes of producing new products is individualization according to the wishes of the client. Conventional methods of production of finished products are often based on serial production, which significantly reduces the price of the product, but also the possibility of individualization of the same. Previous experience in Croatian industry has recognized the opportunity to develop new products, but in order for them to be competitive, it is necessary to have technology that can provide individualization of several hundred products.

Polymer injection molding sets a good starting point. The problem that arises is the fact that a production of a metal mold can be long-lasting process and many times economically unprofitable for the client. By developing materials for additive devices, we come to the finished product. Limitations that occur with additive devices are limited materials, but also mechanical properties with regard to technical materials. The production of molds using additive technology lays the foundation for the production of individualized products that have quality mechanical properties similar to those achieved in molds made of metal. Making polymer molds with additive technology is an economically acceptable and significantly faster process. The problem with molds made with additive technology is their life cycle of up to a maximum of 250 moldings. Previous research has been conducted for the purposes of the doctor thesis. Further research will go in the direction of applying quality protective coatings. This will extend the life cycle of the mold and reduce the cost of making the mold. Individualization towards clients will increase and the impact on the environment will be significantly reduced.

A foundation has been laid for scientific research that will open up new areas in the development of hybrid tools.

\section{REFERENCES}

[1] Stock, T. \& Seliger, G. (2016). Opportunities of Sustainable Manufacturing in Industry 4.0. Procedia CIRP 2016, 40, 536-541. https://doi.org/10.1016/j.procir.2016.01.129

[2] Brozzi, R., Forti, D., Rauch, E., \& Matt, D. T. (2020). The Advantages of Industry 4.0 Applications for Sustainability: Results from a Sample of Manufacturing Companies. Sustainability. https://doi.org/10.3390/su12093647

[3] Sari, T., Gules, H.K., \& Yigitol, B. (2020). Awareness and readiness of Industry 4.0: The case of Turkish manufacturing industry, Advances in Production Engineering \& Management, 15(1), 57-68.

https://doi.org/10.14743/apem2020.1.349
[4] Attaran, M. (2017). The rise of 3-D printing:The advantages of additive manufacturing over traditional manufacturing. Article in Business Horizons. https://doi.org/10.1016/j.bushor.2017.05.011

[5] Polanec, B., Kramberger, J., \& Glodez, S. (2020). A review of production technologies and materials for manufacturing of cardiovascular stents, Advances in Production Engineering \& Management, 15(4), 390-402. https://doi.org/10.14743/apem2020.4.373

[6] Rosnitschek, T., Hueter, F., \& Alber-Laukant B. (2020). FEM-Based Modelling of Elastic Properties and Anisotropic Sinter Shrinkage of Metal EAM. International Journal of Simulation Modelling, 19(2). 197-208. https://doi.org/10.2507/IJSIMM19-2-509

[7] Tehnical application guide: PolyJet For Injection molding, Stratasys, 2016.

[8] Van den Broeck, S. (2017). Guidelines on the design and implementation of stereolithographic $3 D$ printed moulds for low volume injection moulding. Master's dissertation, Ghent University, Belgija.

[9] Bradley, N. (2019). Injection Molding: Process and Design Principles for $3 D$ Printed Molds. The Department of Mechanical and Aerospace Engineering Princeton University.

[10] Simpson, P., Autumn, D., Zakula, J., Nelson, J. K., Dworshak, E. M., \& Johnson, C. A. (2019). Ulven; Injection molding with an additive manufactured tool. Polymer engineering and science. https://doi.org/10.1002/pen.25192

[11] http://www.leolane.com/blog/tessas-weekly-picks-3dprinted-molds/(6/2020)

[12] https://www.makerbot.com/makerbot-replicator-2x/ $(8 / 2020)$

[13] http://3dprintingforbeginners.com/filamentprimer/ (8/2020)

[14] https://www.stratasys.com/(8/2020)

[15] Bačić, I. (2016). Poboljšanje korozijske zaštite nehrđajučeg čelika nanostrukturnim Sol-Gel $\mathrm{ZrO}_{2}$. Doktorski rad, Zagreb.

[16] Owens, D. K. \& Wendt, R. C. (1969). Estimation of the surface free energy of polymers. Journal of Applied Polymer Science, 13(1969), 1741-1747. https://doi.org/10.1002/app.1969.070130815

[17] Wu, S. (1973). Polar and non-polar interactions in adhesion. Journal of Adhesion, 5(1973), 39-55. https://doi.org/10.1080/00218467308078437

[18] Hart, K. R. \& Wetzel, E. D. (2017). Fracture behavior of additively manufactured acrylonitrile butadiene styrene (ABS) materials. Engineering Fracture Mechanics, 177(2017), 1-13. https://doi.org/10.1016/j.engfracmech.2017.03.028

[19] Tiganis, B. E., Burn, L. S, Davis, P., \& Hill, A. J. (2002). Thermal Degradation of Acrylonitrile-Butadiene Styrene (ABS) Blends. Polymer Degradation and Stability, 76(2002), 425-434. https://doi.org/10.1016/S0141-3910(02)00045-9

[20] Liu, W., Kim, H. C., Pak, P. K., \& Kim, J. C. (2006). Effect of Acrylonitrile Content on the Glass Transition Temperature and Melt Index of PVC/SAN Blends. Fibers and Polymers, 7(1), 36-41. https://doi.org/10.1007/BF02933600

[21] Yang, S., Castilleja, J. R., Barrera, E. V., \& Lozano, K. (2004). Thermal analysis of an acrylonitrile-butadiene styrene/SWNT composite. Polymer Degradation and Stability, 83(2004), 383-388. https://doi.org/10.1016/j.polymdegradstab.2003.08.002

[22] Rutkowski, J. V. \& Levin B. C. (1985). AcrylonitrileButadiene-Styrene Copolymers (ABS): Pyrolysis and Combustion Products and Their Toxicity. A review of the literature, U. S. Department of Commerce, Malcolm Baldrige, Secretary National Bureau of Standards, Ernest Ambler 1985. https://doi.org/10.6028/NBS.IR.85-3248

[23] Makhiyanov, N. \& Temnikova, E. V. (2010). Glass Transition Temperature and Microstructure of 
Polybutadienes. Polymer Science, Ser. A, 52(12), 12921300. https://doi.org/10.1134/S0965545X10120072

[24] Billah, K. M. M., Lorenzana, F. A. R., Martinez, N. L., Chacon, S., Wicker, R. B., \& Espalin, D. (2019). Thermal Analysis of Thermoplastic Materials Filled with Chopped Fiber for Large Area 3D Printing Solid Freeform Fabrication, Proceedings of the 30th Annual International Solid Freeform Fabrication Symposium. An Additive Manufacturing Conference, (2019), 892-898.

[25] Reed, T. F., Bair, H. E., \& Vadimsky, R. G. (1974). The causes of pitting and haze on molded ABS plastic surfaces In Recent advances in polymer blends, grafts, and blocks, L. Sperling (Ed.) Springer, Boston, MA, 359-373. https://doi.org/10.1007/978-1-4684-2874-2_15

[26] Mittal, K. L. (1976). Adhesion Measurement of Thin Films. Electrocomponent Science and Technology, 3(1976), 21-42. https://doi.org/10.1155/APEC.3.21

[27] www.fsb.unizg.hr/, N.Štefanić, Uvod u planiranje i analizu pokusa, (2014)

[28] Alvarez, L. F. (2000). Design optimization based on genetic programming. University of Bradford, UK.

[29] Sokolek, M. K. (2007). Utjecaj parametra razvlačnog puhanja na svojstva PET boce. Polimeri, 28(4), Fakultet Strojarstva i Brodogradnje.

\section{Contact information:}

Josip GROŠ, mag.ing.mech

(Corresponding author)

University of applied science Karlovac,

I. Meštrovića 10, 47000 Karlovac, Croatia

E-mail: josip.gros@vuka.hr

Pero RAOS, prof. dr. sc.

University of Slavonski Brod,

Mechanical Engineering Faculty,

Trg I. B. Mazuranic 2, 35000 SlavonskiBrod, Croatia

E-mail: praos@unisb.hr

Mirela LESKOVEC, prof. dr. sc.

University of Zagreb,

Faculty of Chemical Engineering and Technology,

Marulicev trg 19, HR-10000 Zagreb, Croatia

E-mail: mlesko@fkit.hr 